\title{
ARTIGO
}

\section{UMA ANÁLISE DA RESOLUÇÃO DE QUESTÕES SOBRE O TEOREMA DE PITÁGORAS ${ }^{1}$}

\author{
William Vieira ${ }^{2}$ \\ Roberto Seidi Imafuku \\ Emanoel Fabiano Menezes Pereira
}

\begin{abstract}
RESUMO
Apresenta-se, neste artigo, uma análise da resolução de questões sobre o Teorema de Pitágoras de estudantes ingressantes no $1^{\circ}$ ano do Ensino Médio Técnico de uma Instituição Federal de Ensino do Estado de São Paulo. O objetivo dessa investigação foi o de detectar e classificar os principais erros e dificuldades apresentados pelos participantes na identificação e no uso deste teorema. As duas questões, do tipo teste, foram aplicadas para 78 estudantes no início do ano letivo de 2018. A interação de aspectos algorítmicos, intuitivos e formais é o referencial teórico adotado nas análises das resoluções. De maneira geral, verificou-se que os estudantes apresentaram muitas dificuldades no reconhecimento e na aplicação do Teorema de Pitágoras. Como procuramos demonstrar em nossas análises, essas dificuldades estão relacionadas à não interação, por parte dos participantes, de aspectos algorítmicos, intuitivos e formais associados ao uso do teorema.
\end{abstract}

Palavras-chave: Teorema de Pitágoras. Aspectos algorítmicos, intuitivos e formais. Análise de Erros.

\section{INTRODUÇÃO}

Apresentamos, neste artigo, um estudo da resolução de questões que envolvem a identificação, interpretação e aplicação do Teorema de Pitágoras de estudantes ingressantes do Ensino Médio Técnico de uma Instituição Federal de Ensino do Estado de São Paulo. O objetivo dessa investigação é analisar e classificar as dificuldades apresentadas pelos participantes na resolução de problemas sobre o Teorema de Pitágoras, a fim de colaborar com a reflexão coletiva sobre dificuldades de estudantes no estudo deste tema. A análise de erros proposta por Cury (2007) é utilizada na tabulação dos dados e o referencial teórico

\footnotetext{
${ }^{1}$ Como citar este artigo: VIEIRA, William; IMAFUKU, Roberto Seidi; PEREIRA, Emanuel Fabiano Menezes. Uma análise da resolução de questões sobre o Teorema de Pitágoras. ForScience: revista científica do IFMG, Formiga, v. 7, n. 2, e00552, jul./dez. 2019. DOI: 10.29069/forscience.2019v7n2.e552.
}

${ }^{2}$ Autor para correspondência: William Vieira, e-mail: wvieira@ifsp.edu.br. 
adotado na interpretação dos protocolos é a interação de aspectos algorítmicos, intuitivos e formais colocados por Fischbein (1994).

O interesse pelos processos de ensino e de aprendizagem e pelas dificuldades de estudantes com o Teorema de Pitágoras já vem sendo investigados por pesquisadores brasileiros há alguns anos. Em seu trabalho de mestrado, Bastian (2000), elaborou e aplicou uma sequência didática para o ensino deste tema para trinta e seis estudantes do oitavo ano do Ensino Fundamental de uma escola estadual de São Paulo. Um estudo históricoepistemológico-didático sobre o Teorema de Pitágoras e a aplicação e análise de um questionário diagnóstico para estudantes do primeiro ano do Ensino Médio precederam e inspiraram a elaboração da sequência proposta. Segundo a autora, no que diz respeito à aprendizagem do Teorema de Pitágoras, “[...] as atividades constitutivas da sequência parecem ter contribuído para desenvolver nos alunos algumas capacidades, relativamente à aplicação do mesmo como ferramenta para a resolução de problemas" (BASTIAN, 2000, p. 180).

Em seu trabalho de mestrado, Mottin (2004) desenvolveu uma investigação com o objetivo de avaliar o uso de material didático-pedagógico na exploração das relações entre o Teorema de Pitágoras e a Álgebra. Para tal, realizou oito encontros de duas horas-aula (ateliês) com um grupo de dez alunos do oitavo ano do Ensino Fundamental de uma escola particular do Rio Grande do Sul. No primeiro encontro, foi aplicado um questionário para verificar o conhecimento algébrico dos estudantes, por meio do qual se observou que os erros mais frequentes estavam relacionados ao desenvolvimento de produtos notáveis. Nos demais encontros foram desenvolvidos atividades relacionadas ao cálculo de área e ao Teorema de Pitágoras; um novo questionário diagnóstico foi aplicado no último encontro, seguido de entrevistas individuais.

Após a análise do segundo questionário, a autora conclui que “[...] apesar dos erros cometidos por alguns alunos, menos significativos do que os do primeiro consideramos que os ateliês contribuíram para a melhoria da aprendizagem da Álgebra através do Teorema de Pitágoras" (MOTTIN, 2004, p. 81) e, afirma ter notado o desenvolvimento dos estudantes em relação ao início da pesquisa.

Tashima e Silva (2007) responsabilizam o Movimento da Matemática Moderna por distanciar a matemática da vida real e dar pouca importância à geometria por meio da valorização excessiva do simbolismo e de uma terminologia excessiva. Tendo em vista esse problema sobre o ensino de Geometria, as autoras apresentam uma proposta para o ensino do 
Teorema de Pitágoras na perspectiva da resolução de problemas, confecção de materiais didáticos e uso de filmes sobre a História da Matemática.

As atividades propostas pelas autoras foram desenvolvidas em três etapas, com trinta e oito estudantes da primeira série do curso de formação de docentes de Ensino Médio de uma escola estadual do Paraná. Na primeira etapa, foi aplicado um teste de sondagem referente ao conteúdo de Teorema de Pitágoras, quando se verificou que 65,79\% dos alunos foram classificados como ruins, com notas entre zero a três, em dez pontos possíveis. Na segunda etapa, foi aplicada uma sequência didática sobre este tema e, após um período de oito semanas da realização dessas atividades, aplicou-se o mesmo teste de sondagem da primeira etapa, e $63,13 \%$ dos participantes foram classificados como bons, com notas entre sete e dez.

Em suas conclusões, Tashima e Silva (2007) apontam que a sequência de atividades levou os estudantes à percepção da utilidade e da importância da relação pitagórica para além daquela proposta pelos livros didáticos. Além disso, destacam que “[...] as atividades diversificadas contribuíram para desenvolver nos alunos algumas capacidades relacionadas à aplicação do Teorema como ferramenta para a resolução de problemas" (TASHIMA; SILVA, 2007, p. 23).

A fim de avaliar as dificuldades de estudantes no uso do Teorema de Pitágoras, Pereira, Couto e Costa (2016) aplicaram um teste diagnóstico com seis questões sobre esse assunto para vinte alunos do $9^{\circ}$ ano do ensino fundamental de uma escola municipal de Belém do Pará/PA. Uma análise de erros orientou a avaliação qualitativa das respostas dos participantes. Segundo as autoras, as principais dificuldades identificadas estão relacionadas “[...] a falta de compreensão na definição e identificação dos elementos de um triângulo retângulo" (PEREIRA; COUTO; COSTA, 2016, p. 12) além de dificuldades com as operações básicas.

Esses trabalhos colocam em evidência a importância da reflexão sobre os processos de ensino e de aprendizagem, e a avaliação de dificuldades relacionados ao Teorema de Pitágoras. Com o objetivo de colaborar com essas reflexões, nos propomos, neste trabalho, analisar e classificar as dificuldades e os erros cometidos por 78 estudantes ingressantes no Ensino Médio Técnico Federal na resolução de duas questões sobre o Teorema de Pitágoras.

As duas questões avaliadas foram aplicadas para os estudantes no início do ano letivo de 2018 e a atividade ocorreu no campus onde os participantes estudam, por um período de uma hora e meia. Não foi permitido nenhum tipo de consulta a materiais externos e os pesquisadores não ofereceram nenhuma outra orientação, além daquelas contidas nos 
enunciados das questões propostas. As respostas foram classificadas segundo a Análise de Erros proposta por Cury (2007).

A interação de aspectos algorítmicos, intuitivos e formais, colocados por Fischbein (1994), é o referencial teórico adotado nas análises dos protocolos. No que segue, discutimos brevemente essas ideias.

\section{DESENVOLVIMENTO}

\subsection{Referenciais Teóricos}

Fischbein (1994) argumenta sobre a necessidade de observarmos se há ou não a interação de aspectos formais, algorítmicos e intuitivos, quando um sujeito está em atividade matemática. Isto significa olhar a Matemática como um processo criativo e não como um corpo de conhecimentos estruturado e já estabelecido; quando atentamos para este processo criativo, entendemos a Matemática como uma atividade humana, que envolve momentos de “iluminação, hesitação, aceitação e refutação” (FISCHBEIN, 1994).

No que tange aos processos de ensino e de aprendizagem de conceitos e ideias matemáticas, entendemos que a interação desses três aspectos deve guiar nossas escolhas e práticas, se desejamos que nossos estudantes sejam capazes de produzir afirmações matemáticas, construir provas e avaliar, formal e intuitivamente, a validade dessas produções.

$\mathrm{O}$ aspecto formal refere-se aos axiomas, definições, teoremas e demonstrações, que compõem o núcleo das ciências matemáticas e precisam ser considerados quando analisamos o processo de criação em Matemática. Fischbein (1994) reitera que "Axiomas, definições, teoremas e provas têm de penetrar como um componente ativo do processo de raciocínio. Eles devem ser inventados ou aprendidos, organizados, checados e usados ativamente pelo estudante" (FISCHBEIN, 1994). Além disso, aponta que o pensamento proposicional e o uso de construções hipotético-dedutivas não são adquiridos espontaneamente pelos jovens e que somente um adequado processo de ensino pode dar a esses elementos formais características verdadeiramente funcionais.

$\mathrm{O}$ aspecto algorítmico corresponde às técnicas e procedimentos de resolução, que também têm um caráter fundamental no processo de entendimento e criação, pois apenas o conhecimento das estruturas formais (axiomas, definições, teoremas) não é suficiente para conferir habilidades para resolver problemas. Segundo Fischbein, “[...] Esta profunda 
simbiose entre significado e habilidades é uma condição básica para o produtivo e eficiente raciocínio matemático" (FISCHBEIN, 1994).

$\mathrm{O}$ aspecto intuitivo diz respeito a uma intuição cognitiva, um entendimento intuitivo, uma solução intuitiva. A intuição cognitiva é o que um sujeito considera autoevidente e não vê necessidade de prova ou justificação, como afirmações do tipo "A parte é menor que o todo" ou "Multiplicar um número sempre o torna maior". Devido à sua natureza, o conhecimento intuitivo exerce um papel coercivo no raciocínio, definindo caminhos e estratégias para a resolução de problemas. Se estiver de acordo com verdades logicamente justificáveis, pode se tornar um facilitador do processo de conhecimento; caso contrário, torna-se um caminho para contradições e equívocos - como no caso das afirmações apresentadas - e podem se configurar em dificuldades para o processo de aprendizagem.

Cumpre observar que o conceito de intuição não está claramente definido e várias possibilidades de interpretação para este conceito têm sido apresentadas por diferentes autores ao longo dos anos; entretanto, destaca-se, entre essas múltiplas interpretações, a imediatez do conhecimento intuitivo (FISCHBEIN et al., 1981). Entendemos que apresentar uma solução ou interpretação intuitiva para um problema significa utilizar um conhecimento, técnica ou resultado matemático sem que se esteja totalmente consciente de uma justificativa detalhada ou formal. Por exemplo, é fácil aceitar, intuitivamente, que por dois pontos passa uma única reta; por outro lado, temos dificuldade em aceitar que a soma dos ângulos internos de um triângulo é igual a dois ângulos retos.

Sobre o papel que o conhecimento intuitivo desempenha no processo de aprendizagem, Fischbein et al. (1981) alertam que:

O problema de identificar os vieses intuitivos naturais do aluno é importante porque afetam - às vezes de uma maneira muito forte e permanente - seus conceitos, suas interpretações, sua capacidade de compreender, de resolver e de memorizar em uma determinada área. Estamos naturalmente inclinados a manter interpretações que se adequam a esses vieses naturais e intuitivos, e esquecer ou distorcer aqueles que não se encaixam a eles (FISCHBEIN et al., 1981).

Nossa capacidade de processar informações defende Fischbein (1994), não é controlada somente pelas estruturas lógicas, mas também por uma grande quantidade de modelos intuitivos, que agem de maneira implícita, colocando restrições e definindo caminhos para o raciocínio. Ele destaca também que a influência dos modelos intuitivos no pensamento matemático é mais importante e decisiva do que normalmente se acredita e sustenta que essa influência não se restringe a estágios pré-formais do desenvolvimento intelectual do 
indivíduo, mas que permanece agindo no processo de pensamento, mesmo após as estruturas formais do raciocínio estarem plenamente desenvolvidas.

Corbo (2012) apresenta o exemplo da obtenção da fração geratriz de um número racional escrito na forma decimal como uma situação na qual um algoritmo pode negligenciar aspectos formais importantes e desenvolver uma intuição equivocada. A autora analisa o problema de mostrar que a dízima $0,999 \ldots$ é igual a um a partir do procedimento, bastante comum em livros didáticos, de igualar a dízima a x, multiplicar a igualdade por dez e subtrair as duas igualdades. Ao aplicar este algoritmo, estamos supondo que $0,99 \ldots=\frac{9}{10}+\frac{9}{100}+\frac{9}{100}+\frac{9}{1000}+\cdots$ é um número, o que poderia não ser verdade, como é o caso da série harmônica $1+\frac{1}{2}+\frac{1}{3}+\frac{1}{4}+\cdots$. Este aspecto formal do processo é, geralmente, relegado nas explicações apresentadas pelos livros didáticos (CORBO, 2012, p. 101).

Na Questão 1, que propõe a resolução de uma situação-problema, pretendemos avaliar se os estudantes articulam aspectos formais relacionados ao enunciado do Teorema de Pitágoras e à identificação de catetos e hipotenusa, com aspectos intuitivos e algorítmicos presentes no enunciado e na aplicação do teorema. Na Questão 2, que traz um trecho de uma poesia de Millôr Fernandes, procuramos avaliar se os participantes inter-relacionam aspectos formais relativos ao Teorema de Pitágoras com aspectos intuitivos associados à representação em língua natural do enunciado do teorema.

Após as análises de cada uma das questões, apresentamos uma análise conjunta das resoluções, a fim de observar se, e como, os participantes articulam aspectos algorítmicos, intuitivos e formais relacionados ao enunciado e a aplicação do Teorema de Pitágoras.

Estas são as principais ideias e posições que utilizamos nas análises dos dados obtidos nesse trabalho; entretanto, nas análises dos protocolos, a noção de 'contrato didático' aparece com bastante força para alguns dos participantes e, embora este não tenha sido o foco central de nossa investigação, definimos este conceito a seguir e o apontamos nas análises dos dados.

Chama-se contrato didático o conjunto de comportamentos do professor que são esperados pelos alunos e o conjunto de comportamentos do aluno que são esperados pelo professor. Esse contrato é o conjunto de regras que determinam uma pequena parte explicitamente, mas sobretudo implicitamente, do que cada parceiro da relação didática deverá gerir e daquilo que, de uma maneira ou de outra, ele terá de prestar conta perante o outro (BROUSSEAU, 1986, apud SILVA, 2008, p. 50). 
Ainda sobre o 'contrato didático', cumpre destacar que esse tipo de conduta ou comportamento pode afetar o desenvolvimento da aprendizagem dos estudantes e, por isso, entendemos que tal questão merece uma investigação mais aprofundada.

\subsection{Procedimentos metodológicos}

Em nossa investigação, aplicamos questionários diagnósticos com cinco questões para 78 estudantes ingressantes nos $1^{\circ} \mathrm{s}$ anos do Ensino Médio técnico em Mecatrônica (40 alunos) e em Informática (38 alunos) de uma instituição federal de ensino do Estado de São Paulo no ano de 2018. Das cinco questões propostas, três abordam temas de Geometria Plana (Teorema de Tales, cálculo de área e Semelhança de Triângulos) e duas versam sobre o Teorema de Pitágoras. Neste artigo, optamos por discutir as respostas dos participantes para as questões relativas ao Teorema de Pitágoras.

As questões foram resolvidas individualmente, durante um período de uma hora e meia e foram aplicadas no campus em que os participantes estudam, no horário de aula das disciplinas, com autorização prévia da direção da escola. Não foi permitido nenhum tipo de consulta a materiais externos e os pesquisadores não forneceram nenhuma informação adicional além daquelas constantes nos questionários. Os responsáveis pelos participantes assinaram o Termo de Consentimento Livre e Esclarecido (TCLE). Os pesquisadores trabalham na instituição na qual ocorreu a pesquisa, mas não eram professores dos participantes da pesquisa no ano de 2018.

As respostas dos participantes foram classificadas segundo a Análise de Erros (CURY, 2007), procedimento metodológico que está baseado em uma análise de conteúdo, que visa identificar classes de erros que são recorrentes nas resoluções dos problemas propostos. Em seguida, são elaborados quadros que apresentam as frequências e os percentuais que cada tipo de erro identificado ocorreu.

\subsection{Análise dos dados}

A seguir, apresentamos as questões propostas aos estudantes no Questionário Diagnóstico, destacamos os objetivos de cada uma delas e fazemos as análises das respostas dadas pelos participantes. 
Questão 1 - Observe esta figura que representa uma escada apoiada em uma parede. O topo da escada está a $7 \mathrm{~m}$ de altura, e seu pé está afastado da parede $2 \mathrm{~m}$.

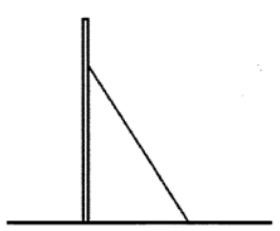

A escada mede, aproximadamente,
(A) $5 \mathrm{~m}$
(B) $6,7 \mathrm{~m}$
(C) $7,3 \mathrm{~m}$
(D) $9 \mathrm{~m}$

Este problema explora o conhecimento dos participantes na aplicação do Teorema de Pitágoras em uma situação-problema. Com esta questão, pretendíamos verificar se os estudantes articulavam aspectos formais relacionados à identificação de catetos e hipotenusa, com aspectos intuitivos e algorítmicos presentes no enunciado e à aplicação do teorema.

A avaliação preliminar dos gabaritos revelou que apenas 35\% dos 78 participantes acertou a Questão 1, número que revela um desempenho abaixo do esperado no uso do Teorema de Pitágoras, uma vez que o problema proposto exigia a identificação dos catetos e aplicação direta do teorema.

A análise das respostas consideradas erradas revelou as classes de erros destacadas no Quadro 1. Cumpre observar que cada resolução pode apresentar mais de um tipo de erro, por isso, a soma dos percentuais não resulta em $100 \%$.

\begin{tabular}{|l|c|c|}
\hline \multicolumn{1}{|c|}{ Descrição do Erro } & Freq & \% \\
\hline $\mathrm{A}_{1}-$ Realizar uma adição ou subtração de $7 \mathrm{~m}$ e $2 \mathrm{~m}$ & 33 & 42 \\
\hline $\mathrm{B}_{1}-$ Não identificar catetos e hipotenusa & 17 & 22 \\
\hline $\mathrm{C}_{1}-$ Erros de cálculo na aplicação do teorema & 8 & 10 \\
\hline $\mathrm{D}_{1}-$ Usar estratégias equivocadas ou desconhecer o teorema & 3 & 4 \\
\hline $\mathrm{E}_{1}-$ Em branco & 7 & 9 \\
\hline
\end{tabular}

Quadro 1- Erros identificados na resolução da Questão 1

Fonte: Elaborado pelos autores.

O Erro tipo $A_{1}$, realizar adição ou subtração de $7 \mathrm{~m}$ e $2 \mathrm{~m}$, foi o mais recorrente nas respostas, tendo ocorrido para $42 \%$ dos participantes. As respostas de José e Maria (Os nomes 
dos estudantes usados neste artigo são fictícios) apresentadas, respectivamente, na Figura 1, são exemplos desse tipo de erro.

Em sua resposta, José não identifica, na figura, os catetos e realiza a soma $7+2=9$, indicando a alternativa (D). Neste caso, fica evidente que o estudante não consegue relacionar o enunciado do problema ao Teorema de Pitágoras, tão pouco consegue relacionar as informações do texto com a figura proposta.
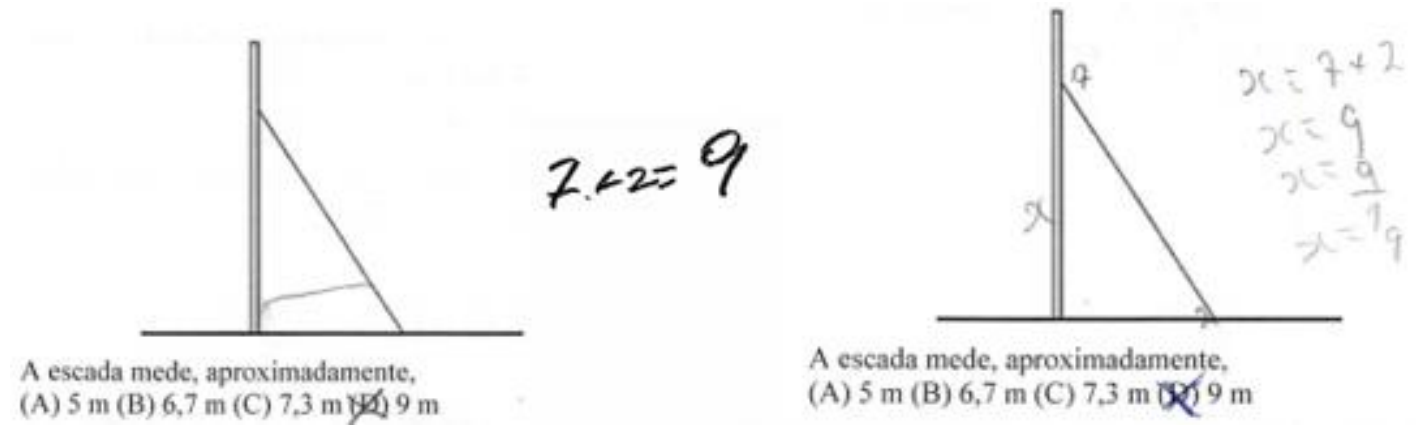

Figura 1 - Respostas de José e Maria para a Questão 1 Fonte: Elaborado pelos autores.

Podemos destacar ainda que, embora tanto José quanto Maria indique desconhecer aspectos formais ligados ao Teorema de Pitágoras, há um imperativo que os faz lançar mão de um algoritmo qualquer, a adição de sete e dois, para fornecer uma resposta. Observe que não se trata apenas de "chutar" uma alternativa para uma questão que não se saiba responder, mas de buscar um cálculo que possa garantir que o problema foi resolvido. Entendemos que esta situação caracteriza uma atuação dos estudantes que seja a esperada pelo professor; ou seja, nestes casos age, implicitamente, o contrato didático, impelindo os estudantes a apresentarem um cálculo qualquer que justifique as decisões tomadas, mesmo eles não tendo entendido do que se trata a questão.

De maneira geral, os $42 \%$ desse tipo erro mostram um desempenho bastante ruim e indicam a ausência de articulação de aspectos algorítmicos, intuitivos e formais relacionados ao Teorema de Pitágoras para estes estudantes.

$O$ Erro tipo $B_{1}$, não identificar catetos e hipotenusa, foi cometido por $22 \%$ dos participantes. As respostas de Pedro e Madalena (Figura 2) são exemplos desse tipo de erro. 


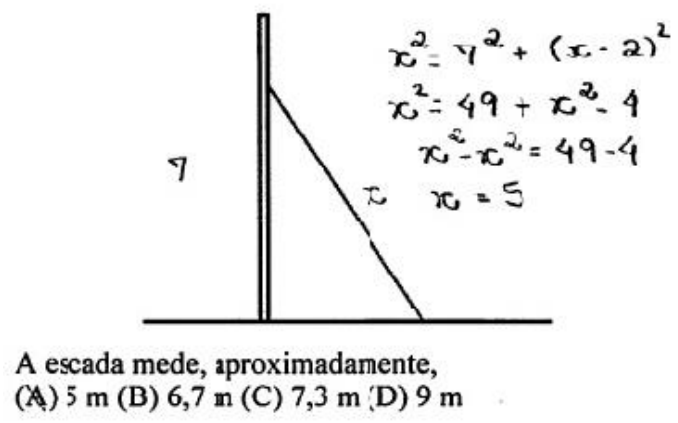

(A) $5 \mathrm{~m}$ (B) $6,7 \mathrm{~m}$ (C) $7,3 \mathrm{~m}$ (D) $9 \mathrm{~m}$

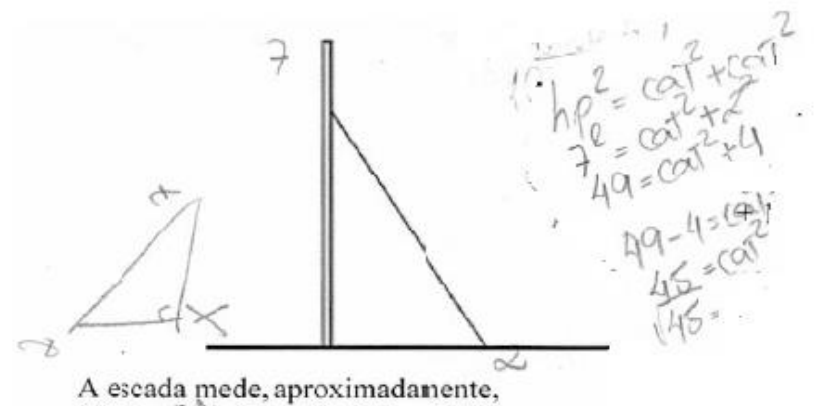

(A) $5 \mathrm{~m}$ (B) $6,7 \mathrm{~m}$ (C) $7,3 \mathrm{~m}$ (D) $9 \mathrm{~m}$

Figura 2 - Respostas de Pedro e Madalena para a Questão 1

Fonte: Elaborado pelos autores.

Em sua resposta, embora não tenha registrado a expressão $\boldsymbol{x}-\mathbf{2}$ na figura, Pedro trata essa diferença como um dos catetos ao aplicar o teorema, o que evidencia uma confusão do estudante com aspectos intuitivos relacionados à interpretação do enunciado e dificuldades com aspectos formais no reconhecimento de elementos envolvidos no Teorema de Pitágoras. Neste caso, a não interação desses aspectos termina por não possibilitar que o estudante tenha êxito na interpretação do enunciado do problema, inviabilizando a resolução.

Além disso, o desenvolvimento de $x^{2}=7^{2}+(x-2)^{2}$ revela dificuldades de Pedro com aspectos algorítmicos relacionados à resolução de equações, ao cálculo algébrico e ao desenvolvimento do quadrado da diferença, temas da Matemática básica. As passagens $(x-2)^{2}=x^{2}-4$ e $x^{2}-x^{2}=49-4$ que leva a $x=5$ são exemplos dessas dificuldades.

Por outro lado, Madalena indica conhecer o Teorema de Pitágoras quando escreve a expressão "hip ${ }^{2}=$ cat $^{2}+$ cat $^{2}$ ", no entanto, não interpretou corretamente os elementos envolvidos no enunciado do problema, pois confunde o cateto de medida $7 \mathrm{~m}$ com a hipotenusa. Além disso, os registros da estudante na figura do problema são confusos e terminam por impedir que ela resolva corretamente a questão.

Neste caso, as dificuldades de Madalena em valer-se de aspectos intuitivos relativos ao movimento de interpretar o enunciado e registrar as informações numéricas na figura proposta a impedem de inter-relacionar os aspectos formais que ela indica conhecer, inviabilizando a resolução do problema.

O Erro tipo $C_{1}$, erros de cálculo na aplicação do teorema, foi cometido por $10 \%$ dos participantes. As respostas de Paulo e Emmy (Figura 3) são alguns dos exemplos identificados nos protocolos. 


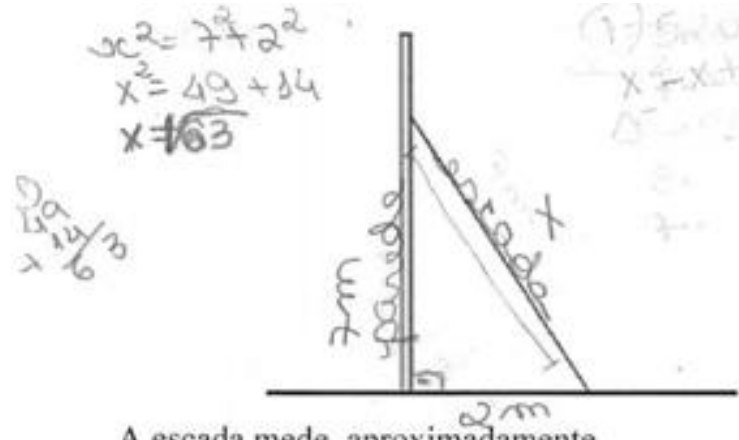

A escada mede, aproximadamente, (A) $5 \mathrm{~m}$ (B) $6,7 \mathrm{~m}$ (C) $7,3 \mathrm{~m}$ (D) $9 \mathrm{~m}$

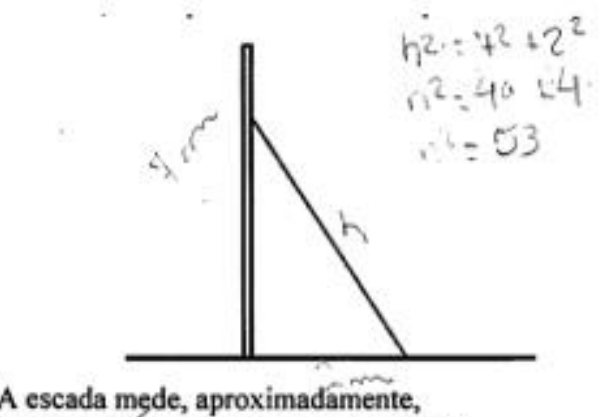

(A) $5 \mathrm{~m}$ (B) $6,7 \mathrm{~m}$ (C) $7,3 \mathrm{~m}$ (D) $9 \mathrm{~m} /$

Figura 3 - Respostas de Paulo e Emmy para a Questão 1

Fonte: Elaborado pelos autores.

Nesses casos, embora os participantes tenham enunciado e aplicado corretamente o Teorema de Pitágoras, se confundiram ao realizar cálculos aritméticos, como no caso de $2^{2}=14$ e a aproximação de 9 para $\sqrt{63}$, no caso de Paulo, e a consideração da aproximação

6,7 para $\sqrt{53}$, no caso de Emmy, perspectivas que evidenciam dificuldades desses estudantes com aspectos algorítmicos.

Além disso, Emmy também apresenta dificuldades com aspectos intuitivos e formais, uma vez que não percebeu que, nas condições do problema, o comprimento da escada não pode ser menor do que altura em que ela está apoiada, e que a hipotenusa não pode ser menor que os catetos.

Apesar desse tipo de erro parecer simples ou que está associado à falta de atenção, a resposta de Emmy indica que a interação, por parte da estudante, de aspectos algorítmicos, intuitivos e formais permitiria a ela identificar e evitar o erro cometido.

O Erro tipo $\mathrm{D}_{1}$, usar estratégias equivocadas ou desconhecer o teorema, foi cometido por $4 \%$ dos participantes e as respostas de João e Luísa (Figura 4) são exemplos desse tipo de erro.

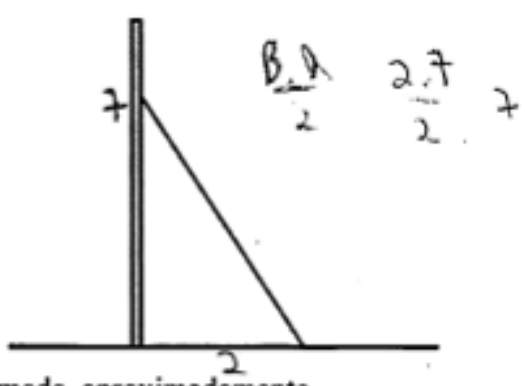

A escada mede, aproximadamente, (A) $5 \mathrm{~m}$ (B) 6,7 m (C) 7,3 m (D) $9 \mathrm{~m}$

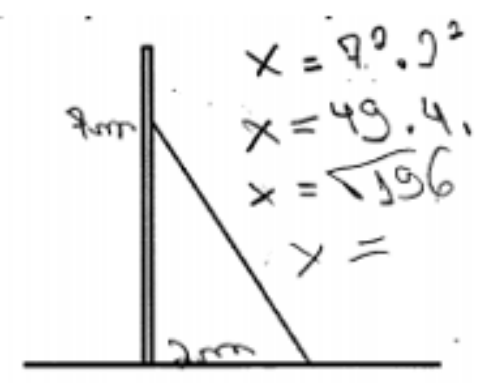

A escada mede, aproximadamente, (A) $5 \mathrm{~m}$ (B) 6,7 m (C) 7,3 m (D) $9 \mathrm{~m}$

Figura 4 - Respostas de João e Luísa para a Questão 1 Fonte: Elaborado pelos autores. 
No caso de João, a estratégia equivocada envolve o uso da fórmula do cálculo de áreas de um triângulo e, apesar de ele tê-la aplicado corretamente, essa resolução evidencia que o estudante não conseguiu, ao ler a questão, identificar a necessidade da aplicação do Teorema de Pitágoras. Essa perspectiva indica dificuldades de João na interação entre aspectos intuitivos, relacionados à leitura e interpretação do enunciado, e aspectos formais relativos ao teorema solicitado.

A resposta de Luíza, por outro lado, nos permite conjecturar que ela tenha tido contato com o Teorema de Pitágoras em algum momento da vida escolar, uma vez que registra corretamente os catetos na figura e os eleva ao quadrado na resolução algébrica; no entanto, a escrita $x=7^{2} \cdot 2^{2}$ mostra dificuldades dessa estudante com aspectos formais relacionados ao teorema. Além disso, a igualdade $x=\sqrt{\mathbf{1 9 6}}$, obtida sem a indicação do quadrado na linha anterior, indica dificuldades com aspectos algorítmicos relacionados à resolução de equações incompletas do $2^{\circ}$ grau.

Esse tipo de erro, embora tenha ocorrido para um número pequeno de estudantes, indica que um tipo de ensino que não promova a interação de aspectos algorítmicos, intuitivos e formais tende a desenvolver um tipo de conhecimento estéril, que não cumpre a tarefa de tornar os aprendizes capazes de interpretar e resolver problemas com destreza. Os 9\% de participantes que deixaram a Questão 1 em branco (Erro tipo E1) ajudam a corroborar essa perspectiva.

Apresentamos a seguir a análise da Questão 2.

Questão 2 - Millôr Fernandes, em uma bela homenagem à Matemática, escreveu um poema do qual extraímos o fragmento a seguir:

Às folhas tantas de um livro de Matemática, um Quociente apaixonou-se um dia doidamente por uma Incógnita.

Olhou-a com seu olhar inumerável e viu-a do ápice à base: uma figura ímpar;

olhos romboides, boca trapezoide, corpo retangular, seios esferoides.

Fez de sua uma vida paralela à dela, até que se encontraram no infinito. 
"Quem és tu?", indagou ele em ânsia radical.

"Sou a soma do quadrado dos catetos.

Mas pode me chamar de Hipotenusa."

(Millôr Fernandes. Trinta Anos de Mim Mesmo.)

A Incógnita se enganou ao dizer quem era. Para atender ao Teorema de Pitágoras, deveria dar a seguinte resposta:

(A) "Sou a soma dos catetos. Mas pode me chamar de hipotenusa."

(B) "Sou o quadrado da soma dos catetos. Mas pode me chamar de hipotenusa."

(C) "Sou o quadrado da soma dos catetos. Mas pode me chamar de quadrado da hipotenusa."

(D) "Sou a soma dos quadrados dos catetos. Mas pode me chamar de quadrado da hipotenusa."

Com a Questão 2 buscamos avaliar se os participantes inter-relacionam aspectos formais relativos ao Teorema de Pitágoras com aspectos intuitivos associados à representação em língua natural do enunciado.

A avaliação preliminar das respostas revelou que $41 \%$ dos 78 participantes acertaram a Questão 2, ao assinalar o item (D). Após a análise dos erros dessa questão, apresentamos uma análise comparativa dos acertos das Questões 1 e 2.

Como a Questão 2 não requer uma solução algorítmica, consideramos cada uma das alternativas incorretas como um erro distinto. O Quadro 2 traz os percentuais envolvidos nas respostas erradas.

\begin{tabular}{|l|c|c|}
\hline \multicolumn{1}{|c|}{ Descrição do Erro } & Freq & \% \\
\hline $\mathrm{A}_{2}$ - "Sou a soma dos catetos. Mas pode me chamar de hipotenusa" & 24 & 31 \\
\hline $\begin{array}{l}\mathrm{B}_{2} \text { - "Sou o quadrado da soma dos catetos. Mas pode me chamar de } \\
\text { hipotenusa" }\end{array}$ & 10 & 13 \\
\hline $\begin{array}{l}\mathrm{C}_{2} \text { - "Sou o quadrado da soma dos catetos. Mas pode me chamar de } \\
\text { quadrado da hipotenusa" }\end{array}$ & 8 & 10 \\
\hline $\mathrm{D}_{2}$ - Em branco & 4 & 5 \\
\hline
\end{tabular}

Quadro 2 - Erros identificados na resolução da Questão 2

Fonte: Elaborado pelos autores. 
De maneira geral, todos os tipos de erros do Quadro 2 indicam um desconhecimento dos participantes sobre aspectos formais relacionados ao enunciado do Teorema de Pitágoras.

O Erro tipo $\mathrm{A}_{2}$, "Sou a soma dos catetos. Mas pode me chamar de hipotenusa", o mais frequente entre os participantes, nos parece um tipo de dificuldade mais profunda, pois a afirmação é bastante diferente do enunciado do teorema, uma vez que sequer menciona o uso das potências (quadrados). Cabe observar que o alto percentual desse erro está em consonância com o Erro tipo $\mathrm{A}_{1}$, da Questão 1, "Realizar uma adição ou subtração de 7m e $2 \mathrm{~m}$ ", que ocorreu para $41 \%$ dos participantes. Esses tipos de erros $\left(\mathrm{A}_{1}\right.$ e $\left.\mathrm{A}_{2}\right)$ indicam a ausência de conhecimento de aspectos formais relacionados ao Teorema de Pitágoras para estes estudantes.

Os erros $\mathrm{B}_{2}$ e $\mathrm{C}_{2}$ indicam uma confusão dos participantes entre as expressões "quadrado da soma" e "soma de quadrados". Esse tipo de dificuldade está associado à não interação de aspectos algorítmico-intuitivos e formais relacionados à tradução de sentenças algébricas, uma vez que admitem que $(a+b)^{2}=a^{2}+b^{2}$, o que indica uma percepção intuitiva equivocada sobre essas ideias. No caso do erro $\mathrm{B}_{2}$, há o agravante de não se considerar o quadrado da hipotenusa.

A escolha dessas afirmações por $23 \%$ dos participantes indica que para esses estudantes permanecem muitas dúvidas e confusões sobre conceitos e técnicas da álgebra básica, como, por exemplo, os produtos notáveis. Entendemos que um tipo de ensino que não favorece a interação de aspectos algorítmicos, intuitivos e formais pode ser apontado como um dos responsáveis pela permanência dessas dificuldades.

\subsection{Uma reflexão sobre os acertos identificados nas duas questões}

A avaliação individual das questões revelou que 35\% dos participantes acertou a Questão 1 e $41 \%$ a Questão 2, porém, embora possa se atribuir alguma consonância a esses números, a análise mais detalhada dos protocolos mostra que $27 \%$ dos estudantes acertaram ambas. O fato de $14 \%$ dos participantes terem acertado somente a Questão 2, indica que apenas o conhecimento de aspectos intuitivos e formais, relacionados à interpretação correta do enunciado do teorema em língua natural, não é suficiente para conferir aos estudantes habilidade em resolver problemas.

De maneira análoga, o fato de 8\% dos estudantes terem acertado a Questão 1 e errado a Questão 2, aponta que somente o conhecimento de aspectos algorítmicos e intuitivos, 
associados a aplicação do teorema, não é suficiente para conferir o conhecimento de aspectos formais relacionados ao Teorema de Pitágoras.

Essas situações evidenciam que a não interação de aspectos algorítmicos, intuitivos e formais pode dificultar o processo de aprendizagem, posição defendida por Fischbein (1994).

\section{CONSIDERAÇÕES FINAIS}

De maneira geral, consideramos o desempenho dos participantes nas questões que abordaram o Teorema de Pitágoras, muito abaixo do que seria esperado para ingressantes no Ensino Médio. Avaliamos a situação como grave, uma vez que este é um tema bastante relevante do Ensino Fundamental e que é considerado conhecimento prévio para vários conceitos e áreas da Matemática, como, por exemplo, no estudo da Geometria Espacial e Geometria Analítica.

Os índices de $42 \%$ do erro tipo $A_{1}$, "Realizar uma adição ou subtração de 7 m e 2 m" e os $31 \%$ do erro $\mathrm{A}_{2}$, "Sou a soma dos catetos. Mas pode me chamar de hipotenusa", são evidências desse desempenho ruim e indicam que muitos dos participantes possuem pouco ou nenhum tipo de conhecimento sobre o Teorema de Pitágoras. As respostas de João e Maria (Figura 1) são exemplos da profundidade desses tipos de dificuldades. Além disso, identificamos dificuldades dos participantes relacionadas a álgebra básica, como produtos notáveis, perspectiva também colocada por Mottin (2004).

$\mathrm{Na}$ análise das respostas, identificamos uma possível influência do contrato didático estabelecido entre os participantes da pesquisa e seus professores no Ensino Fundamental, manifestado na orientação de que não deveriam deixar questões sem respostas, o que pode ter levado alguns dos participantes a apresentar um cálculo qualquer envolvendo os dados do problema, mesmo sem compreender o que deveriam fazer.

Nesse sentido, concordamos com a posição de Tashima e Silva (2007), quando destacam que, ao não perceber a relação da geometria com a realidade em que vive, os jovens não conseguirão desenvolver plenamente seu pensamento geométrico.

Conforme procuramos defender ao longo de nossas análises, um tipo de ensino que não promove a interação de aspectos algorítmicos, intuitivos e formais dos conceitos e ideias matemáticas pode ser apontado como um dos responsáveis pelos altos índices de fracasso nas questões analisadas. 


\title{
AN ANALYSIS OF THE RESOLUTION OF QUESTIONS ABOUT THE PYTHAGOREAN THEOREM
}

\begin{abstract}
This article presents an analysis of the resolution of questions about the Pythagorean Theorem of freshman students in the 1st year of the Technical High School of a federal teaching institution in the state of São Paulo. The objective of this investigation was to detect and classify the main errors and difficulties presented by the participants in the identification and use of this theorem. The two questions, of the test type, were applied to 78 students at the beginning of the academic year of 2018. The interaction of algorithmic, intuitive and formal aspects is the theoretical reference adopted in the analysis of the resolutions. In general, it was verified that the students presented many difficulties in the recognition and the application of the Pythagorean Theorem. As we try to demonstrate in our analyzes, these difficulties are related to the participants' noninteraction of algorithmic, intuitive and formal aspects associated to the use of the theorem.
\end{abstract}

Keywords: Pythagorean theorem. Algorithmic, intuitive and formal aspects. Error Analysis.

\section{REFERÊNCIAS}

BASTIAN, Irma Verri. O Teorema de Pitágoras. 2000. 229 f. Dissertação (Mestrado em Educação Matemática) - Pontifícia Universidade Católica de São Paulo, São Paulo, 2000.

CORBO, Olga. Um estudo sobre os conhecimentos necessários ao professor de Matemática para a exploração de noções concernentes aos números irracionais na educação básica. 2012. 313 f. Tese (Doutorado em Educação Matemática) - Universidade Bandeirante de São Paulo, São Paulo, 2012.

CURY, Helena Noronha. Análise de erros: o que podemos aprender com as respostas dos alunos. 1. ed. Belo Horizonte: Autêntica, 2007.

FISCHBEIN, Efraim; TIROSH, Dina.; MELAMED, U. Is it possible to measure the intuitive acceptance of a mathematical statement? Educational Studies in Mathematics, Dordrecht: Kluwer Academic Publisher, n. 12, p. 491-512, 1981.

FISCHBEIN, Efraim. The interaction between the formal, the algorithmic, and the intuitive components in a mathematical activity. In Rolf Biehler et al. (Org.) Didactics of Mathematics as a Scientific Discipline, Dordrecht: Kluwer Academic Publisher, p. 328-375. 1994.

MOTTIN, Elisandra. A Utilização de material didático-pedagógico em ateliês de matemática para o estudo de Teorema de Pitágoras. 2004. 117 f. Dissertação (Mestrado em Ciências e Matemática) - Pontifícia Universidade Católica do Rio Grande do Sul, Porto Alegre, 2004. 
PEREIRA, Mayara Gabriella Grangeiro; COUTO, Ana Paula Nascimento Pegado; COSTA, Acylena Coelho. Análise de erros em questões de Teorema de Pitágoras: um estudo com alunos do Ensino Fundamental. São Paulo: 2016.

SILVA, Benedito A. Contrato Didático. In: MACHADO, Silvia D. A. (Org.), Educação matemática: uma (nova) introdução. São Paulo: EDUC. p. 49-75. 2008.

TASHIMA, Marina Massaco; SILVA, Ana Lúcia da. As lacunas no ensino-aprendizagem da geometria. Disponível em:

http://www.gestaoescolar.diaadia.pr.gov.br/arquivos/File/producoes_pde/artigo_marina_mass aco_tashima.pdf. Acesso em: 03 set. 2018.

\section{DADOS DOS AUTORES}

\section{William Vieira}

E-mail: wvieira@ifsp.edu.br

Currículo Lattes: http://lattes.cnpq.br/6106510148543215

Doutor em Educação Matemática pela Universidade Anhanguera de São Paulo, mestrado e licenciatura em Matemática pelo Instituto de Matemática e Estatística da Universidade de São Paulo. Professor do Instituto Federal de São Paulo (IFSP) Campus Guarulhos. Membro do Grupo de Estudos e Pesquisa em Educação Matemática e Formação de Professores (GEPEMFOP do IFSP) - Campus Guarulhos.

\section{Roberto Seidi Imafuku}

E-mail: roberto.imafuku@ifsp.edu.br

Currículo Lattes: http://lattes.cnpq.br/7926295090638853

Doutor em Educação Matemática pela Universidade Anhanguera de São Paulo, mestrado acadêmico em Educação Matemática pela Pontifícia Católica (PUC). Professor do Instituto Federal de São Paulo (IFSP) Campus Guarulhos. Membro do Grupo de Estudos e Pesquisa em Educação Matemática e Formação de Professores (GEPEMFOP do IFSP) - Campus Guarulhos.

\section{Emanoel Fabiano Menezes Pereira}

E-mail: emanoel.pereira@ifsp.edu.br

Currículo Lattes: http://lattes.cnpq.br/4601893345601275

Mestre em Matemática pela Universidade Federal do ABC (UFABC) Campus Santo André, especialista em Educação à Distância pela Universidade Federal Fluminense (UFF) e graduado em Licenciatura em Matemática pela Universidade de São Paulo (USP). Atualmente é professor efetivo do Instituto Federal de Educação, Ciência e Tecnologia de São Paulo (IFSP), é orientador e preceptor do Programa de Residência Pedagógica - área de Matemática do IFSP/Campus Guarulhos. 